\title{
Analysis of different brands Ayurvedic Drug (Tamra Bhasma) by Non Destructive Technique using NaI(Tl) Scintillation Detector
}

\author{
Ashwini A ${ }^{1}$, B.R. Kerur ${ }^{2 *}$ \\ ${ }^{1}$ Department of Physics, Gulbarga University, Kalaburagi, Karnataka-585106 \\ ${ }^{2}$ Controller of Examination, Central University of Karnataka, Kadaganchi, Kalaburagi, Karnataka-585367 \\ *Corresponding Author: kerurbrk@gmail.com, Tel.: + Tel.: +919448213160
}

Available online at: www.isroset.org

Received: 30/Jan/2019, Accepted: 16/Feb/2019, Online: 28/Feb/2019

\begin{abstract}
In the present work ayurvedic drug Tamra bhasma (TB) of different brands were procured, the pellets of different thickness of TB was prepared, by employing the non destructive technique, X-ray mass attenuation coefficients of Tamra bhasma have been measured using the variable energy X-ray source $\mathrm{Am}^{241}$ as a source of characteristic X-rays of low energy $(17.781,22.581,32.890$ and $44.216 \mathrm{keV})$. These X-rays were used for interaction process with the sample (Tamra bhasma) and incident and transmitted photons are collected by a $\mathrm{NaI}(\mathrm{Tl})$ scintillation X-ray detector. The mass attenuation coefficients values are determined by following all procedure such as counting times, background intensities, error involved in counting statistics and good geometry is maintained for the counting of photon beams. The experimentally obtained values are compared with the theoretically calculated values, using the WinXcom data program. From obtained results, it reveals that, the variation in percentage deviation (PD) of experimental and theoretical calculated values of mass attenuation coefficient corresponds to the amount of bioenhancers, incorporated in Tamra bhasma during preparation process. Here variation in percentage deviation reflects drugs as the admixture of elements other than the pharmaceutical active ingredients (here in Tamra bhasma $\mathrm{Cu}$ is the Active Pharmaceutical Ingredient) which focuses on the purity and also change in quality of medicines.
\end{abstract}

Keywords-Ayurvedic drug, X-ray Mass attenuation coefficient, Tamra bhasma, NaI(Tl) scintillation/X-ray Detector

\section{INTRODUCTION}

The use of photon interaction has been increased in various fields like medical, agriculture, industry, defense, radiation shielding etc, hence the knowledge of absorption and scattering of X-ray/gamma-ray has become a necessary and important field of research [1] The accurate value of attenuation coefficient of X-ray or $\gamma$-rays in various different materials are necessary for many fields, like biological, medical, agricultural, environmental and industrial field, etc., the parameter mass attenuation coefficient (symbolically indicated as $\mu / \rho)$ is important in determining the radiation parameters like effective atomic number, mass energy absorption coefficient, total electronic cross section, total atomic cross-section, molecular cross-section, X-ray fluorescence, etc. The knowledge of physical parameter such as mass attenuation coefficient $(\mu / \rho$ in $\mathrm{cm} 2 / \mathrm{g})$ is useful in understanding physical properties of any element and material in compound form for characterizing the sample. In the literature review there are number of works are available on both experimental and theoretical Mass attenuation coefficient $(\mu / \rho)$ of X-ray or $\gamma$-rays [2-4]. Hubbell (1982) [5] has published the data tables of mass attenuation coefficients and mass energy-absorption coefficients for photon energy ranging from $1 \mathrm{keV}$ to $20 \mathrm{MeV}$ for 40 elements, 45 mixtures and compounds of $Z=1$ to 92 . The XCOM program (1987), for calculation of theoretical mass attenuation coefficient $(\mu / \rho)$ developed by Berger and Hubbell [6], was modified as WinXcom by the author Gerward $[7,8]$ and now WinXcom programme have been used by every researchers for theoretical calculation of X-ray attenuation coefficients and interaction cross sections in materials [9]. By following earlier research work, we have conducted the work on analysis of the ayurvedic medicine Tamra bhasma from different brands, by measuring the mass attenuation coefficient experimentally and calculating the theoretical values for comparison and validating the experimental values, to get the information on purity of Tamra bhasmas. No literature is found on mass attenuation coefficient of ayurvedic bhasmas, since for the first time the work is carried out by using the low energy $\mathrm{X}$-rays by the help of $\mathrm{NaI}(\mathrm{Tl})$ scintillation detector.

\section{RELATED WORK}

The experimental method for an accurate measurement of mass attenuation coefficient was earlier developed [10] and 
concluded that the best values of $\mu / \rho$ can be obtained by thickness which lie in the transmission (T) range $0.5 \geq \mathrm{T} \geq$ 0.25 . By measuring the $X$-ray mass attenuation coefficient at different energy, effective atomic and electron numbers, and atomic and electronic cross sections in $\mathrm{Ti}, \mathrm{Ni}$, and their alloys have been calculated [11] Mass attenuation coefficient for some natural minerals quartz (1101), Quartz (1100), Quartz (0001), Orthoclase (010), Orthoclase (100), Gypsum, Pyrite, and Pyroxene at 22.1, 25.0, 59.5, and 88.0 $\mathrm{keV}$ Photon energies have been experimentally measured and compared theoretically with WinXcom data [12] and shown that the $\mu / \rho$ can change with variation in atomic number and electronic number for different compositions alloys. Diagnostic point of view by the mass attenuation coefficient, effective atomic number and electron density of dosimetric material had been calculated [13]. We have reported earlier mass attenuation coefficient of mono and disaccharides for photons in the energy range of 5-15 keV [14] and also for ten different mono (Glucose, fructose, mannose) and disaccharides (Sucrose, lactose and maltose) in the energy range from 8 to $32 \mathrm{keV}$ incident photon energy [15] using $\mathrm{NaI}(\mathrm{Tl})$ scintillation detector also measured the mass attenuation coefficients in medicinal plants in low energy range $8-32 \mathrm{keV}$ revealing that the method used helps in determining the uniformity of elemental content of samples from different regions $[16,17]$. The extensive study on an effective atomic number in composite materials of a known composition such as bronze, brass, Perspex, soldering material, Bakelite and rare earth samples as lead-tin alloys by various researchers were reported, using mass attenuation coefficient as primary data $[18,19]$. In biological and geological samples mass attenuation coefficient have been determined experimentally and reported the difference in attenuation properties of samples, a difference of $47 \%$ for plants samples and $22 \%$ of a difference in animal tissues, in the 7-12 keV energy range [20]. X-rays mass attenuation coefficient for different barite concrete used in radiation protection as shielding against ionizing radiation was measured, and reflected the light on the difference in the experimental and theoretical values of mass attenuation coefficients, due to the variations in the chemical composition of the samples and the trait of the mixing rule [21]. Experimentally, the radiation parameter such as mass attenuation coefficient, total attenuation cross section, total electronic cross section, mass energy absorption coefficient and CT number are evaluated at $81,122,356$ and $511 \mathrm{keV}$, gamma photon energy for studying the energy dependence of radiation interaction parameters of some organic compounds [22]. One of the author said that we should have the knowledge of X-ray production cross section, in order to get the exact quantity of element[23]

In the literature, no experimental data is available on the study of herbo-mineral compounds or Ayurvedic bhasmas medicines/drugs materials. Further, also the quality test of the medicinal drug is usually carried out by destructive testing method but as such, no literature data is available for test the quality of the drug by non-destructing methods. The purpose of the present study is to determine the X-ray mass attenuation coefficient of 'Tamra bhasma(TB)' an Ayurvedic drug in the low energy region 17.781, 22.581, 32.890 and $44.216 \mathrm{keV}$ using transmission method and compared data theoretically through the program WinXcom. Hence, the present work describes experimental determination of Mass attenuation coefficient of X-rays, using $\mathrm{NaI}(\mathrm{Tl})$ Scintillation detector following the procedures set by Creagh and Hubbell [22] by selecting ayurvedic medicine Tamra Bhasma (TB) of four brands, as attenuating material. Tamra bhasma is a preparation of Copper $(\mathrm{Cu})$ and some herbal ingredient resulting a smooth and fine powder. By using the determined mass attenuation coefficient values the quality of the drug is tested and analyzed for the implication values.

The selected Tamra Bhasma is useful in treatment of various diseases; it is used in the treatment of Anemia, loss of appetite, acidity as per the Baidyanth brand manufacture (BTB). And in the treatment of Abdominal disorders, ascites, edema and respiratory disorders as per Patanjali manufacture (PTB) and by the Vyas brand product (VTB) it is consumed in case of having problem with Hichki (Hiccups), Aamatisar (Diarrhoea), Yakratvikar (Liver disorder), Visham jwar (Chronic fever), Aamlapitt (Hyperacidity) and lastly Dhootapapeshwar brand (DTB) product has mentioned as per direction of physician on Tamra bhasma pack/box.

To get information of mass attenuation coefficient values of Ayurvedic drug sample Tamra bhasma(TB), at low photon energy, and for comparative analysis of the purity of TB,s the present work has been carried out

\section{MATERIALS AND MeTHODS}

\section{Experimental Details}

When X-rays or photons interact with matter its intensity progressively decreases, due to absorption and scattering of primary photon in the material media. When a beam of mono-energetic photons intensity $I_{o}$ interacts or penetrates through an absorbing material of thickness ' $x$ ' and density ' $\rho$ ' respectively, than reduces the intensity as ' $I$ '. The process can be mathematically expressed by Beer-Lambert's law as below

$$
\frac{I}{I_{o}}=\exp \left[-\left(\frac{\mu}{\rho}\right) x\right]
$$

The equation is rewritten as

$$
\frac{\mu}{\rho}=-\frac{1}{x} \ln \left(\frac{I}{I_{o}}\right)
$$

Where, $\mathrm{I} / \mathrm{I}_{\mathrm{o}}$ give the transmission fraction of intensity for the applied incident photon energy, $(\mu / \rho)$ is the mass attenuation coefficient for element under consideration. For any compound or homogeneous mixture the $\mathrm{X}$-ray mass attenuation coefficient $(\mu / \rho)$ is estimated by Bragg's 
additivity law or it is also known as mixture rule, is mathematically written as

$$
\frac{\mu}{\rho}=\sum \omega_{i}\left(\frac{\mu}{\rho}\right)_{i}
$$

$(\mu / \rho)_{i}$ is the mass attenuation coefficient of the $i^{\text {th }}$ element in unit of $\mathrm{cm}^{2} / \mathrm{gm}$ and $\omega_{\mathrm{i}}$ is the fraction by weight of the $\mathrm{i}^{\text {th }}$ element in $\mathrm{mg}$.

In the present study, four different brands Tamra Bhasam were purchased and their details are mentioned in table 1.

The $\mathrm{X}$-ray spectrometer i.e., $\mathrm{NaI}(\mathrm{Tl})$ detector was calibrated using the variable energy X-ray source and standardization method is adopted as described [10] to determine the mass attenuation coefficient $(\mu / \rho)$ of X-rays. The good geometry experimental setup used here is shown in figure 1 and is similar to the one described by us earlier in the literature [24].

The experiment for determining mass attenuation coefficient is carried out by using variable energy X-Ray (VEX) source $\mathrm{Am}^{241}(10 \mathrm{mCi})$ as the main source of excitation radiation with four different targets (Mo, $\mathrm{Ag}, \mathrm{Ba}$, and $\mathrm{Tb}$ ) for producing the fluorescent X-Rays with characteristic energies 17.781, 22.581, 32.890 and $44.216 \mathrm{keV}$ respectively. The Block diagram of the experimental setup is shown in Figure 1. Photons from variable energy X-Ray source (S), passed through the Collimator (C1) and were incident on the specimen (A) under examination kept normal to incident photon beam, then the beam transmits through the second collimator $(\mathrm{C} 2)$ and reaches the $\mathrm{NaI}(\mathrm{Tl}) \mathrm{X}$-ray detector (D). A bicron-made integrated assembly of $25 \mathrm{~mm}$ dia $\mathrm{x} 4 \mathrm{~mm}$ thick $\mathrm{NaI}(\mathrm{Tl})$ scintillation detector is mounted on a photomultiplier tube (PMT) served as the x-ray detector. Oxford model PCAP plus PC plug-in single PCI card, had on board high voltage supply, pre-amplifier, amplifier, and $1 \mathrm{k}$ ADC. The detectors parameters were set through the PC control mechanism. The transmitted photon spectrum was recorded with the help of a $\mathrm{PC}$ based Multi-channel analyzer (MCA) using the OXWIN MCA software. Collimator C1 and $\mathrm{C} 2$ collimate the incident and transmitted beams respectively. The spectrum recorded by Multichannel Analyzer (MCA) consist transmitted X-Rays and because of the poor energy resolution of the detector, the weighted average energy of $\mathrm{K}_{\alpha}$ and $\mathrm{K}_{\beta}$ is estimated and compared with measured X-ray mass attenuation coefficients. The mass attenuation coefficient is measured by selecting the photon intensity area under the peak i.e., FWHM is taken as transmitted photo peak since it is the average weighted energy of $\mathrm{K}_{\alpha}$ and $\mathrm{K}_{\beta}$ energies. The spectrometer is standardized for the standard elements like Magnesium ( $\mathrm{Mg})$, Aluminium ( $\mathrm{Al})$, Copper $(\mathrm{Cu})$ and lead $(\mathrm{Pb})$ element. After the standardization of spectrometer study samples tamra bhasma were used and obtained experimental results of mass attenuation coefficient of samples (Tamra Bhasma) are presented in Table 2.

\section{RESUlTS AND DiscuSSION}

The mass attenuation coefficient values for the pure elements were measured experimentally and theoretically and presented in the Table 1 which shows a good agreement between the theoretical values and experimental values. The method adopted for the measurement of attenuation coefficients, for photon intensity, is selected under the FWHM of the photo peak and Shapiro(1948) counting statistics is maintained [25], the sample is kept mid way between the detector and source, the conditions of Creagh and Hubbell (1987) [26] and also M Nagabhushan [24] conditions are adopted. Hence the results are agreeing within $2 \%$ with the theoretical values taken from WINXCOM values, which shows the method adopted is accurate and gives good results and can be adopted for any materials for the determination of mass attenuation coefficient values. So, this method is applied to determine the mass attenuation coefficients of Ayurvedic Drugs. The experimental values of ' $\mu / \rho$ ' were obtained by obeying the Beer-Lambert's law using the transmitted intensity of incident photon, and the slope or inclination of the plot of neperian logarithm (ln) of transmitted intensity (I/Io) as a function of Photon energy, gives mass attenuation coefficient $(\mu / \rho)$ of X-rays [20] thus the attenuation coefficients were calculated by the method of least squares fit.

Experimentally determined mass attenuation coefficient values for four brand Tamra bhasma (TB), viz., Baidyanth Tamra bhasma (BTB), Dhootapapeshwar Tamra bhasma (DTB), Patanjali Tamra bhasma (PTB) and Vyas Tamra bhasma (VTB), presented in Table 2. In Table 2 first column represents sample code or brand name of Tamra bhasma, second column represents the X-ray/photon energy range used in experiment, third column indicates the experimental mass attenuation coefficient $(\mu / \rho)$ values with error obtained as experimental results under study, fourth column indicates the theoretical values of mass attenuation coefficient calculated by WinXcom program for comparison of the experimental results. The fifth column represents the Percentage Deviation (PD) between theoretical and experimental $(\mu / \rho)$ values. The PD varies from 18 to $86 \%$ which gives the information about the experimental values have deviated from the theoretical values

For good agreement between experimental and theoretical values about $4 \%$ of PD is acceptable, such as, about $1-2 \%$ of uncertainty involves in theoretical values, since the reproducibility of experimental values is within $2 \%$ and about $2 \%$ of error is due to contribution from the counting statistics, aerial density, thickness measurements are taken in account, but above $4 \%$ of $\mathrm{PD}$ should be considered as disagreement between theoretical and experimental values. From Table 2, PD is varying from 33- 
$83 \%$ in low energy region, and $49-86 \%$ in high energy region, in the present work PD occurred is more than $4 \%$, here $\mu / \rho$ values by WinXCom program are due to outcome of active drug component ie., it is copper $(\mathrm{Cu})$ in $\mathrm{TB}$, which is known as active pharmaceutical ingredient (API), whereas the experimental $(\mu / \rho)$ values are resultant of overall constituents in the drug Tamra bhasma (TB), i.e., in Ayurveda pharmacopeia, bioenhancers are the substances commonly included in the drug formulations in order to enhance the bioavailability of drugs [27]. Drug or nutrient in combination with a bioenhancers substance provides more availability of drug thereby reducing the amount of active molecule that is required by drug [28].

In present work from our experimental results, values in Table 2, reveals the least value of PD corresponds to less amount of bioenhancers/enhancers incorporated in drug/bhasma (means in Tamra bhasma along with copper/Tamra other elements are incorporated) preparation or Small PD indicates the drug is having Active Pharmaceutical Ingredient in significant amount and very less quantity of inactive pharmaceutical ingredient or bioenhancers. And highest value of PD indicates the higher amount of bioenhancers added in drug/bhasma preparation. So from present study, it reveals that the Dhootapapeshwar Tamra bhasma (DTB) has the lesser bioenhancers with the active pharmaceutical ingredient or drug/nutrient $(\mathrm{Cu})$. And the Patanjali Tamra bhasma (PTB) has the highest value of bioenhancers with drug/nutrient $(\mathrm{Cu})$. Therefore the variation in experimental mass attenuation coefficient can be because of the bioenhancers present in Tamra bhasma in all brands, which affects on the change in the quality of drug/bhasma from one manufacturer to other manufacture, thus it impacts on the purity and quality of bhasma or medicine. Thus Ayurvedic drugs or bhasmas of any element such as $\mathrm{Cu}, \mathrm{Fe}$, and $\mathrm{Sn}$ are made of admixtures of herbs and minerals in varying proportions. They incorporates with some low $\mathrm{z}$ elements such as $\mathrm{H}, \mathrm{C}, \mathrm{O}$, and $\mathrm{N}$ as drug (as inactive ingredient components) and acts as an accelerator for ingestion of the medicine. Hence PD is found to be different in Tamra Bhasma prepared by different manufacturers. The present work tells that the quality and purity of the drug can be checked by knowing the mass attenuation coefficient values of the bhasmas by comparing different brand samples.

\section{CONClusion AND FUture SCOPE}

It has been noticed that the experimental mass attenuation coefficient of ayurvedic Tamra bhasma measured at low photon energies are smaller than the theoretical values for all the four brand Tamra bhasma. The present work has been undertaken, to get the information about the purity of the drug tamra bhasma from different manufacturer, through the obtained percentage deviation values, one can predict the purity of any sample, a non destructive method adopted here to measure the $\mu / \rho$ is very efficient technique to measure the $\mu / \rho$ value accurately. Present work point out that, the nondestructive technique is effective tool in drug analysis. To our best knowledge the experimental $\mu / \rho$ values for herbo -mineral formulations/ Ayurvedic medicines are not available in the literature. This work points out here about the extent purity of elemental content of the ayurvedic medicines, further work is needed in order to know, the complete characterization of the Ayurvedic/Herbo-mineral bhasma. It is worthy to emphasize here that by using the adopted method one can accurately measure $\mu / \rho$ for any material even with a low-resolution detector and can predict the purity of compounds or samples.

\section{REFERENCES}

[1] V. V. Awasarmol, "Gamma ray attenuation parameters of inorganic nonlinear optical materialsin the energy range $122 \mathrm{keV}$ to $1330 \mathrm{keV}$ ', Indian Journal of Pure and Applied Physics, Vol. 55, No.01, pp.65-72, 2017.

[2] S. R. Manohara, S. M. Hangodimath, "Studies on effective atomic numbers and electrn densities of essential amino acids in the energy range $1 \mathrm{keV}-100 \mathrm{GeV}$ ', Nuclear Instruments and Methods in Physics Research Section B: Beam Interaction with Materials and Atoms, Vol.258, Issue.2. pp-321-328, 2007.

[3] S. R. Manohara, S. M. Hangodimath, L. Gerward, "The effective atomic numbers of some biomolecules calculated by two methods: A comparative study", Medical Physics, vol.36, Issue.1, pp.137141, 2009.

[4] B. Akca \& S. Z. Erzeneoglu, "The Mass Attenuation Coefficients, Electronic, Atomic, and Molecular Cross Sectins, Effective Atomic Numbers, and Electron Densities for Compounds of Some Biomedically Important Elements at $59.5 \mathrm{keV}$ ', Science and Technology of Nuclear Installations, Vol. 2014 (2014) pages 8

[5] J. H. Hubbell, "Photon Mass Attenuation Coefficient and Energyabsorption Coefficients from $1 \mathrm{keV}$ to $20 \mathrm{MeV}$ ", International Journal of Applied Radiation Isotope, Vol.33, pp.1269-1290, 1982.

[6] M. J. Berger, J. H. Hubbell, "XCOM: Photon cross sections on a personal computer", NBSIR,87-3597, 1987.

[7] L. Gerward, N. Guilbert, K. B. Jensen, H. Levring, "X-ray absorption in mater. Reengineering XCOM", Radiation Physics and Chemistry, Vol.60, Issue.1-2, pp.23-24, 2001.

[8] L. Gerward, N. Guilbert, K. B. .Jensen H. Levring, "WinXcom-a program for calculating X-ray attenuation coefficiens", Radiation Physics and Chemistry, Vol.71, Issue.3-4, pp.653-654, 2004.

[9] M. W. Marashdeh, I. F. Al-Hamarneh, Eid. M. A. Munem, A. A. Tajuddin, A. Ariffin, S. Al-Omari, "Determining the mass attenuation coefficient, effective atomic number, and electron density of raw wood and binderless particleboards of Rhizophora spp. By using Monte Carlo simulation", Results in Physics,. Vol.5, pp.228-234, 2015.

[10] B. R. Kerur, S. R. Thontadarya, B. Hanumaiah, "A Novel Method for the Determination of X-Ray Mass Attenuation Coefficients", Applied. Radiation and Isotopes, Vol.42, No.6, pp.571-575, 1991.

[11] I. Han, L. Demir, "Mass attenuation coefficients, effective atomic and electron numbers of $\mathrm{Ti}$ and $\mathrm{Ni}$ alloys", Radiation Measurements, Vol.44, Issue 3, pp.289-294, 2009.

[12] I. Han, L. Demir, M. Sahin, "Determination of mass attenuation coefficients, effective atomic and electron numbers for some natural minerals", Radiation Physics and Chemistry Vol.78, Issue. 9, pp.760-764, 2009.

[13] S. B. Kaginelli, T. Rajeshwari, Sharanabasappa, B.R. Kerur, S. Anilkumar, "Effective atomic numbers and electron density of 
dosimetric material", Journal of Medical Physics, Vol.34, No.3, pp.176-179, 2009.

[14] Chitralekha, B. R. Kerur, M. T. Lagare, R. Nathuram, D. N. Sharma, "Mass attenuation coefficients of saccharides for lowenergy X-rays", Radiation Physics and Chemistry,Vol.72, Issue.1,pp.1-5, 2005.

[15] B. R. Kerur, V. T. Manjula, M. T. Lagare S. Anilkumar, "Mass attenuation coefficient of saccharides for $X$-rays in the energy range from $8 \mathrm{keV}$ to $32 \mathrm{keV}$ ', Radiation Measurements, Vol.44, Issue.1, pp.63-67, 2009.

[16] R. B. Morabad, B. R. Kerur, "Mass attenuation coefficients of Xrays in different medicinal plants", Applied Radiation and Isotopes, Vol.68, Issue.2, pp.271-274, 2010.

[17] S. S. Teerthe, B. R. Kerur, "X-Ray Mass Attenuation Coefficient of Medicinal Plant Using Different Energies $32.890 \mathrm{keV}$ to $13.596 \mathrm{keV}$ ", In the Materials Today proceedings International Conference on Materials Research and Applications ICMRA -2016 (11-13th March 2016), Department of Physics, CMR Technical Campus, India, Vol. 3 Issue 10, Part B, pp.3925-3929, 2016.

[18] M. P. Singh, A. Sharma, B. Singh, B.S. Sandhu, "A non-destructive technique for assigning effective atomic number to scientific samples by scattering of $59.54 \mathrm{keV}$ gamma photons", Nuclear Instruments and Methods in Physics Research A, Vol. 619, Issue 1-3, pp.63-66, 2010.

[19] M. P. Singh, B. S. Sandhu \& B. Singh, "Measurement of the effective atomic number of composite materials using Rayleigh to Compton scattering of $279 \mathrm{keV}$ gamma rays", Phyica Scripta, Vol.76, No.4, pp.281-286, 2007.

[20] V. Trunova, A. Sidorina, V. Kriventsov, "Measurement of X-ray mass attenuation coefficients in biological and geological samples in the energy range of 7-12 keV', Applied Radiation and Isotopes, Vol.95, No.1, pp.48-52, 2015.

[21] A. A. T. Junior, M. S. Nogueira, V. Vivolo, M. P. A. Potiens, L. L. Campos, "Mass attenuation coefficients of $X$-rays in different barite concrete used in radiation protection as shielding against ionizing radiation", 2nd International Conference on Dosimetry and its Applications (ICDA-2) University of Sirrey, Guildford, United Kingdom,3-8 July 2016. Radiation Physics and Chemistry, Vol.140, No.11, pp.349-354, 2017.

[22] M. Singh, A. Tondon, B. S. Sandhu, B. Singh, "Energy dependence of radiation interaction parameters of some organic compounds", Radiation Physics and Chemistry, Vol.145, No.4, pp.80-88, 2018.

[23] P.K. Prajapati, "Effect of Re-Correction in the Recoil Energy on $K$ and L-shell X-ray Production Cross-Section by Proton Induced", International Journal of Scientific Research in Physics and Applied Sciences, Vol.6, Issue.3,pp.35-39, 2018.

[24] N. M. Nagabhushan, B. R. Kerur, M. T. Lagare, R. Nathuram, M.C. Abani, "Technique for Measurement of Photon Intensity for the Determination of $\mu \mathrm{m}$ in the Low Photon Energy Region", Journal of x-ray Science Technology, Vol.12, No.3, pp.161-167, 2004.

[25] M. E. Rose, M. M. .Shapiro, "Statistical Error in Absorption Experiments", Physical Review, Vol.74, No.12, pp.1853-1864, 1948.

[26] D. C. Creagh, J. H. Hubbell, "Problems Associated with the Measurement of X-ray Attenuation Coefficients. I. Silicon Report on the International Union of Crystallography X-ray Attenuation Project", Acta Crystallographica Section A, Vol.43, pp.102-112, 1987.

[27] S. Mukkavalli, V. Chalivendra, B. R. Singh, "Physico-chemical analysis of herbally prepared silver nanoparticles and its potential as a drug bioenhancers", OpenNano. Vol.2, pp.19-27, 2017.

[28] N. Atal and K. L. Bedi, "Bioenhancers: Revolutionary concept to market", Journal of Ayurveda and Integrative Medicine, Vol.1, No.2, pp.96-99, 2010.

\section{AUTHORS PROFILE}

Dr. B. R. Kerur M.Sc, Ph.D Physical Science from Karnataka University Dharwad. He is serving as a professor of Physics since September 2010. currently working asController of Examination (COE) at Central University of Karnataka, Kadaganchi, Kalaburagi, Karnataka-585367, India. He has published more than 100 research papers in reputed international journals including Thomson Reuters (SCI \& Web of Science) and conferences including IEEE and it's also available online. His main research work focuses radiation dose measurement, radiation monitoring, and he is life member of 15 academic bodies, also worked as executive member of the Indian Association for the Radiation Protection (IARP) during the period 2011-13.

Miss Ashwini A. is currently pursuing Ph.D. under the guidance of Prof. B. R. Kerur, at department of Physics, Gulbrag University, Kalaburagi-585106, Karnataka. She is working on the low energy X-ray Interaction of Raditiation with matter.

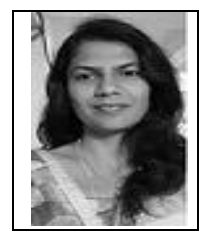

